\title{
Design Pattern Classifiers under Attack for Security Evaluation using Multimodal System
}

\author{
Rupali B. Navalkar \\ ME Second Year (CSE) \\ H.V.P.M's COET, S.G.B.Amravati University, \\ Amravati, India
}

\author{
Prof. Rajeshri R. Shelke \\ Assi Prof. (CSE),ME(CSE) \\ H.V.P.M's COET, S.G.B.Amravati University, \\ Amravati, India
}

\section{ABSTRACT}

Pattern classification systems based on machine learning algorithms are commonly used in securityrelated applications like biometric authentication, network intrusion detection, and spam filtering, to discriminate between a "legitimate" and a "malicious" pattern class., in which data can be purposely manipulated by humans to undermine their operation. As this adversarial scenario is not taken into account by classical design methods, pattern classification systems may exhibit vulnerabilities, whose exploitation may severely affect their performance, and consequently limit their practical utility. Pattern classification theory and design methods to adversarial settings. Here, propose a framework for empirical evaluation of classifier security that formalizes and generalizes the main ideas proposed in the literature, and give examples of its use in real applications. Reported results show that security evaluation can provide a more complete understanding of the classifier's behaviour in adversarial environments, and lead to better design choices. This framework can be applied to different classifiers on one of the application from the spam filtering, biometric authentication and network intrusion detection. So in this propose an algorithm for the generation of training and testing sets to be used for security evaluation. Now result shows providing security to system using application as blogger for this applying spam filtering, biometric authentication methods. That shows pattern classification for detecting spam comments which easy to detect spam. Multimodal System means that using different inputs system will stored the data for testing and training generation for provide highly security.

\section{KEYWORD}

Pattern Classification, Security Evaluation, Adversarial application, Spam Detection, Spoofing Attack.

\section{INTRODUCTION}

Security evaluation of pattern classifiers under attack describes pattern classification systems problems because of different attacks.

Pattern classification system are based on machine learning algorithms are commonly used in securityrelated applications like biometric authentication, network intrusion detection, and spam filtering. Contrary to traditional ones, these Applications have an intrinsic adversarial nature since the input data can be purposely manipulated by an intelligent and adaptive adversary to undermine classifier operation. This often gives rise to an arms race between the adversary and the classifier designer. examples of attacks against pattern classifiers are: submitting a fake biometric trait to a biometric authentication system (spoofing attack); modifying network packets belonging to intrusive traffic to evade intrusion detection systems (IDSs); manipulating the content of spam emails to get them past spam filters (e.g., by misspelling common spam words to avoid their detection) .

It is now acknowledged that, since pattern classification systems based on classical theory and design methods do not take into account adversarial settings, they exhibit vulnerabilities to several potential attacks, allowing adversaries to undermine their effectiveness. A systematic and unified treatment of this issue is thus needed to allow the trusted adoption of pattern classifiers in adversarial environments. A framework is used for evaluation of classifier security that formalizes and generalizes the 


\section{www.ijtsrd.com}

training and testing datasets. Results show that security evaluation can provide a more complete understanding of the classifier's behaviour in adversarial environments, and lead to better design choices. Adversarial machine learning is a research field that lies at the intersection of machine learning and computer security. In the previous sections, we introduced the problem of pattern recognition and adversarial classification.

In Design pattern classification under attack using a framework for empirical evaluation of classifier security that formalizes and generalizes the main ideas proposed in the literature, and give examples of its use in real applications. Reported results show that security evaluation can provide a more complete understanding of the classifier's behavior in adversarial environments, and lead to better design choices. This framework can be applied to different classifiers on one of the application from the spam filtering, and network intrusion detection. Considering Multimodal system is the security of account when one of the modes is successfully spoofed. that the proposed methodology to rank score fusion rules is capable of providing correct ranking of score fusion rules under spoof attack .Spoofing attacks where one person or program purposely falsifying data and there by gaining an illegitimate advantage. It discusses how the classical design cycle of pattern classifiers should be revised to take security into account.

\section{LITERATURE REVIEW}

In this review on previous work, Security Evaluation of Pattern classification systems based on machine learning algorithms are commonly used in securityrelated applications like biometric authentication, network intrusion detection, and spam filtering, to discriminate between a "legitimate" and a "malicious" pattern class[1][2]. Pattern classification systems based on classical theory and design methods do not take into account adversarial settings. They exhibit vulnerabilities to several potential attacks, allowing adversaries to undermine their effectiveness [3]. Biometric systems have been found to be useful tools for person identification and verification.

A biometric characteristic is any physiological of behavioural trait of a person that can be used to distinguish that person from other people [2][6].
Spoof attacks consist in submitting fake biometric traits to biometric systems [2][4], and this is a major threat in security.

The presence of an intelligent and adaptive adversary makes the classification problem highly nonstationary[1], and makes it difficult to predict how many and which kinds of attacks a classifier will be subject to during operation, that is, how the data distribution will change. In particular, the testing data processed by the trained classifier can be affected by both exploratory and causative attacks, while the training data can only be affected by causative attacks, In both cases, during operation, testing data may follow a different distribution than that of training data, when the classifier is under attack. Therefore, security evaluation cannot be carried out according to the classical paradigm of performance evaluation [1][2][3].

Security problems often lead to a "reactive" arms race between the adversary and the classifier designer [2]. At each step, the adversary analyzes the classifier defences, and develops an attack strategy to overcome them [1]. Many authors implicitly performed security evaluation as a what-if analysis, based on empirical simulation methods; they mainly focused on a specific application, classifier and attack, their goal was either to point out a previously unknown vulnerability, or to evaluate security against a known attack.

\section{A. Previous Review On Security Evaluation}

Many authors implicitly performed security evaluation as a what-if analysis, based on empirical simulation methods; however, they mainly focused on a specific application, classifier and attack, and devised ad hoc security evaluation procedures based on the exploitation of problem knowledge and heuristic techniques. Their goal was either to point out a previously unknown vulnerability, or to evaluate security against a known attack.

\section{PROPOSED WORK}

In this proposed work we address issues above by developing a framework for the empirical evaluation of classifier security at design phase that extends the model selection and performance evaluation steps of the classical design cycle. We summarize previous 
work, and point out three main ideas that emerge from it. We then formalize and generalize them in our framework.

- To pursue security in the context of an arms race it is not sufficient to react to observed attacks, but it is also necessary to proactively anticipate the adversary by predicting the most relevant, potential attacks through a what-if analysis; this allows one to develop suitable countermeasures before the attack actually occurs, according to the principle of security by design.

- To provide practical guidelines for simulating realistic attack scenarios, we define a general model of the adversary, in terms of her goal, knowledge, and capability, which encompass and generalize models proposed in previous work.

- Since the presence of carefully targeted attacks may affect the distribution of training and testing data separately, we propose a model of the data distribution that can formally characterize this behavior, and that allows us to take into account a large number of potential attacks;

- Also propose an pattern classifier for security evaluation, which can naturally accommodate application-specific and heuristic techniques for simulating attacks.

\section{A. Framework}

In this work we create a new framework to provide security to adversarial application. we address issues developing a framework for the empirical evaluation of classifier security also propose algorithm used for security evaluation and generation of training and testing sets. when user wants to enter into the system first user $\log$ in into the system if user is a authenticated person and he enter correct user id, password and also correct pattern only then system permit user to access the system. If enter pattern is wrong then pattern manager manage or classify the pattern and provide more security the user account. And also provide account information to user. In the proposed system pattern manager find out the pattern of attack and provide security to user account before attack is completed. We define a general models :-

\section{Modules:}

Attack Scenario and Model of the Adversary

$>$ Pattern Classification
$>$ Adversarial classification

$>$ Security modules

The definition of attack scenarios is ultimately an application-specific issue, it is possible to give general guidelines that can help the designer of a pattern recognition system.

Pattern classification is the scientific discipline whose goal is to classify the objects into a number of classes or categories, depending on the type of application, these objects may be any type of measurements, images or signal waveforms that need to be classified.

In pattern classification, typically a set of patterns (the raw data), whose class is unknown, is given. In addition, proper actions can be taken based on the outcome of the pattern classification. a classifier is designed by training it on a set of patterns (samples or feature vectors) whose true class is known referred also as training set or design set, to find a classification function.

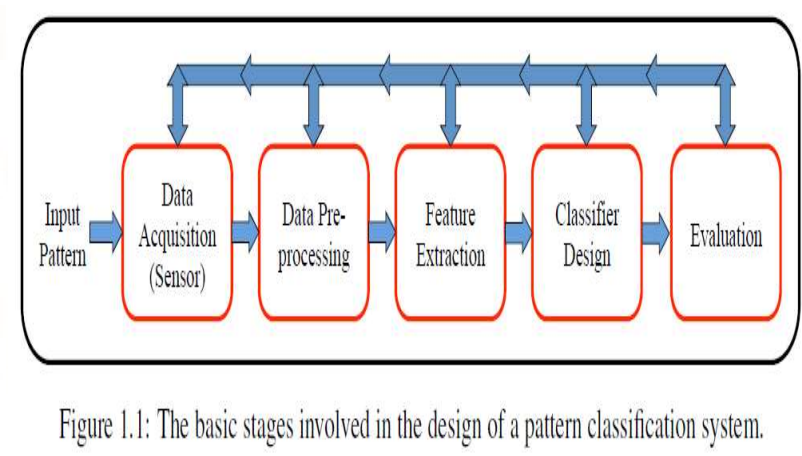

Figure 1.1 shows the various stages followed for the design of a pattern classification system. The first step is to collect a pre-processed set of training samples.

The role of data pre-processing module is therefore to segment the pattern of interest from the background, remove noise and any other operation which will contribute in defining a compact representation of the pattern. Features are then extracted from each training sample. In practice, a larger than necessary number of feature candidates is generated and then the best of them are adopted. The classifier, which is chosen among different algorithms, is trained on appropriate features. Finally, once the classifier has been designed (trained), one can evaluate the performance of the designed classifier. 


\subsection{Objectives.}

2. Our future work will be devoted to develop techniques for simulating attacks for different applications.

3. Prevents developing novel methods to assess classifier security against these attacks.

4. The presence of an intelligent and adaptive adversary makes the classification problem highly non-stationary.

5. We also propose an algorithm for the generation of training and testing sets to be used for security evaluation, which can naturally accommodate application-specific and heuristic techniques for simulating attacks.

\section{B. System Architecture}

The main goal is difficult to represent to escape the design classifiers in the Adversarial Classification Problems with the help of the framework. An artifice for providing the security for classifier designer is to mask the data to the Adversary. In the case of Armsrace, it is not possible to recommend how many and what type of attacks a classifier will incur during operation, the classifier security should proactively evaluate using a what-if analysis, by simulating potential attack scenarios. The primary goal is to formulate or model the adversary as the optimization of an actual function. The effective simulation of attack scenarios requires a formal model of the adversary. In many cases, according to the knowledge of classifier and capability of manipulation of data, the adversary acts rationally to attain a goal of security evaluation.

Our main goal is to provide a quantitative and general-purpose basis for the application of the whatif analysis to classifier security evaluation, based on the definition of potential attack scenarios. Our main goal is to provide a quantitative and general-purpose basis for the application of the what-if analysis to classifier security evaluation, based on the definition of potential attack scenarios.

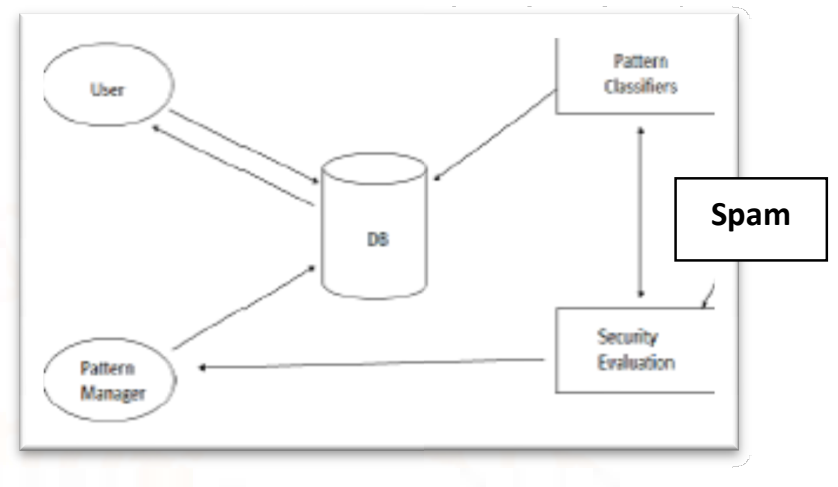

Figure 2. System Architecture

\section{SYSTEM DESIGN}

It shows that how to intruder trying to penetrate system using different source which try to harm system or manipulate information which is not detecting by system. To avoid this problems system using different applications that are set of algorithms which detect unusual traffic and spamming. System trying to provide security through applications that intruder trying to attacks.

Below shows the intruder to legitimate system diagram that easy to understand. 


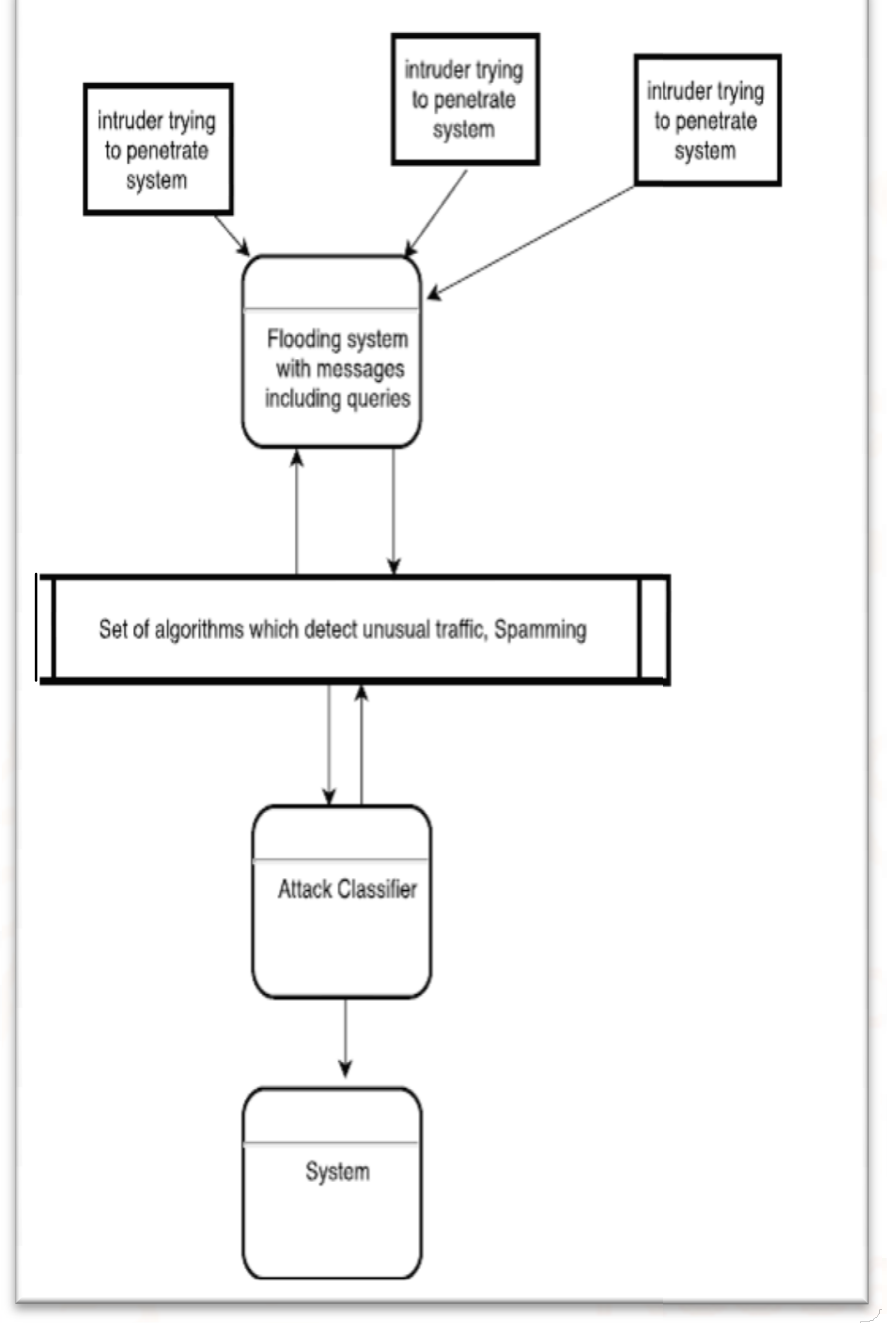

Figure 3. Intruder to legitimate system

This is system diagram that shows are intruder trying to penetrate to system and how to system detect this using spamming, and unusual traffic. Using attack classifiers concept our system using spam filters and biometric simulation methods to become strong system.

\section{Execution Details}

This section presents the detailed information about the classification techniques in data mining in order to demonstrate the complete process. Firstly, after starting the system shown to the user is as follows. In our system a new concept added i.e. Multimodal System. So this mean that using different inputs system will stored the data for testing and training generation for provide highly security. User can sign in using different inputs password that using biometric simulation as password in that it choose image as password in sign in using face and in sign in it takes as password as basic password on home page, system using blog for performing operation. Blogger is a website that displays postings views, thoughts. A person who keeps a web $\log ($ blog), An online diary, a personal chronological log of thoughts published on a web page, also called web log.

Spam filter is a program that is a program that is used to detect unsolicited and unwanted data. And prevent this using blog in our blog users posts comments that comments are detecting by admin.

Admin has to authority to check comments and using the knowledge he decides that comments will approved or reject. Because of this it prevents our system from vulnerabilities also admin can guide to users for comments that why this comments approved or reject. Then admin and users both can add new post on blog.

For security evaluation our system used IP address concept it means when user post comments on blog from their systems, it will stored their system IP address from this IP address we can find malicious users that will help in future.

\section{CONCLUSION}

In this Paper our main contribution is a framework for empirical security evaluation that formalizes and generalizes ideas from previous work, and can be applied to different classifiers, learning algorithms, and classification tasks. It is grounded on a formal model of the adversary, and on a model of data distribution that can represent all the attacks considered in previous work, provides a systematic method for the generation of training and testing sets that enables security evaluation; application-specific techniques for attack simulation. This is a clear advancement with respect to previous work, since without a general framework most of the proposed techniques could not be directly applied to other problems. By combining multiple sources of information, these systems improve matching performance, also the paper focused on innovative security evaluation of pattern classifiers that deployed in adversarial environments and analyzing on security evaluation of pattern classification under attack applying various methods. 


\section{ACNOWLEDGEMENT}

I feel deeply indebted and thankful to all who opined for technical knowhow and helped in collection of data. also feel thankful to my guide shelke mam and to all who directly and indirectly help me for transactional information. A special thanks to my family members for constant support and motivation.

\section{REFERENCES}

[1] Battista Biggio, Member, IEEE, Giorgio Fumera, Member, IEEE, and Fabio Roli, Fellow, IEEE ,“ Security Evaluation of Pattern Classifiers under Attack", IEEE TRANSACTIONS ON KNOWLEDGE AND DATA ENGINEERING, VOL. 26, NO. 4, APRIL 2014.

[2] S.P.Mohana Priya[1], S.Pothumani, "Identifying Security Evaluation of Pattern Classifiers Under attack", International Journal of Innovative Research in Science, Engineering and Technology (An ISO 3297: 2007 Certified Organization)Vol. 4, Issue 3, March 2015.

[3] Kale Tai. , Prof. Bere S. S., "A Survey on: Security Evaluation of Pattern Classifiers under Attack", International Journal of Innovative Research in Computer and Communication Engineering (An ISO 3297: 2007 Certified Organization) Vol. 3, Issue 11, November 2015.

[4]Yadigar Imamverdiyev, Lala Karimova, Vugar Musayev , James Wayman, “TESTING BIOMETRIC SYSTEMS AGAINST SPOOFING ATTACKS", The Second International Conference "Problems of Cybernetics and Informatics" September 10-12, 2008, Baku, Azerbaijan.

[5] Tanisha Aggarwal, Dr. ChanderKant Verma, "Spoofing Technique for Fingerprint Biometric system" ,IJSRD - International Journal for Scientific Research \& Development| Vol. 2, Issue 03, 2014.

[6] Arun Ross and Anil K. Jain,"MULTIMODAL BIOMETRICS: AN OVERVIEW" , Appeared in Proc. of 12th European Signal Processing Conference (EUSIPCO), (Vienna, Austria), pp. 1221-1224, September 2004.
[7] R.N. Rodrigues, L.L. Ling, and V. Govindaraju, "Robustness of Multimodal Biometric Fusion Methods against Spoof Attacks," J. Visual Languages and Computing, vol. 20, no. 3, pp. 169-179, 2009.

[8] R. R. Shelke ,Dr. V. M. Thakare, Dr. R . V. Dharaskar,"Study of Data Mining Approach for Mobile Computing Environment",International Journal on Computer Science and Engineering (IJCSE), ISSN : 0975-3397, Vol. 4, 12 Dec 2012 ,pp.1920-1923

[9] Dr. Amitabh wahi, C.Prabhakaran "A Literature Survey on Security Evaluation of Pattern Classifiers under Attack" International Journal of Advance Research in Computer Science and Management Studies Volume 2, Issue 10, October 2014. 